\title{
Completeness-resolvable graphs
}

\author{
Min Feng ${ }^{1}$ Xuanlong $\mathrm{Ma}^{2 *}$ Huiling $\mathrm{Xu}^{1}$ \\ ${ }^{1}$ School of Science, Nanjing University of Science and Technology, Nanjing, 210094, China \\ ${ }^{2}$ School of Science, Xi'an Shiyou University, Xi'an, 710065, China
}

\begin{abstract}
Given a connected graph $G=(V(G), E(G))$, the length of a shortest path from a vertex $u$ to a vertex $v$ is denoted by $d(u, v)$. For a proper subset $W$ of $V(G)$, let $m(W)$ be the maximum value of $d(u, v)$ as $u$ ranging over $W$ and $v$ ranging over $V(G) \backslash W$. The proper subset $W=\left\{w_{1}, \ldots, w_{|W|}\right\}$ is a completeness-resolving set of $G$ if

$$
\Psi_{W}: V(G) \backslash W \longrightarrow[m(W)]^{|W|}, \quad u \longmapsto\left(d\left(w_{1}, u\right), \ldots, d\left(w_{|W|}, u\right)\right)
$$

is a bijection, where

$$
[m(W)]^{|W|}=\left\{\left(a_{(1)}, \ldots, a_{(|W|)}\right) \mid 1 \leq a_{(i)} \leq m(W) \text { for each } i=1, \ldots,|W|\right\} .
$$

A graph is completeness-resolvable if it admits a completeness-resolving set. In this paper, we first construct the set of all completeness-resolvable graphs by using the edge coverings of some vertices in given bipartite graphs, and then establish posets on some subsets of this set by the spanning subgraph relationship. Based on each poset, we find the maximum graph and give the lower and upper bounds for the number of edges in a minimal graph. Furthermore, minimal graphs satisfying the lower or upper bound are characterized.
\end{abstract}

Key words: completeness-resolvable, resolving sets, distance, edge coverings, bipartite graphs

2010 MSC: 05C12, 05C35, 05C70, 05C75

\section{Introduction}

For a positive integer $m$, denote by $[m]$ the set of positive integers at most $m$, and for a positive integer $k$, write

$$
[m]^{k}=\left\{\left(a_{(1)}, \ldots, a_{(k)}\right) \mid a_{(i)} \in[m] \text { for all } i \in[k]\right\} .
$$

Throughout of this paper, a graph means a finite and simple graph with at least two vertices. Given a graph $G$, we always use $V(G)$ and $E(G)$ to denote the vertex and

\footnotetext{
*Corresponding author.

E-mail address: fgmn_1998@163.com (M. Feng), xuanlma@mail.bnu.edu.cn (X. Ma), xuhuiling@njust.edu.cn (H. Xu).
} 
edge sets of $G$, respectively. The order and size of $G$ are the cardinalities of $V(G)$ and $E(G)$, respectively. We say that $G$ is connected if for any vertices $x, y \in V(G)$, there is a path from $x$ to $y$ in $G$. The distance between $x$ and $y$, denoted by $d(x, y)$, is the length of a shortest path from $x$ to $y$. For a proper subset $W$ of $V(G)$, write

$$
m(W)=\max \{d(w, u) \mid w \in W, u \in V(G) \backslash W\} .
$$

The proper subset $W=\left\{w_{1}, \ldots, w_{|W|}\right\}$ of $V(G)$ is a resolving set of $G$ if

$$
\Psi_{W}: V(G) \backslash W \longrightarrow[m(W)]^{|W|}, \quad u \longmapsto\left(d\left(w_{1}, u\right), \ldots, d\left(w_{|W|}, u\right)\right)
$$

is an injection.

Resolving sets of a graph were first introduced, by Slater [10] and independently, by Harary and Melter [6] in the 1970s. Subsequently, various applications of resolving sets have appeared in the literature, as diverse as network discovery and verification [2], robot navigation [8], pharmaceutical chemistry [4], strategies for the Mastermind game [5], combinatorial optimization [9] and so on. For an overview of resolving sets and related topics, we refer to [1], [3] and [7].

A resolving set $W$ of a connected graph $G$ is a completeness-resolving set if $\Psi_{W}$ is a bijection. We say that $G$ is completeness-resolvable if $G$ admits a completenessresolving set. Note that every connected graph has a resolving set.

Problem 1. Which graphs are completeness-resolvable?

A vertex $x$ of a graph $G$ is universal if $x$ is adjacent to every other vertices in $G$. Denote by $\mathcal{K}$ the set of graphs which have a universal vertex. Let $\mathcal{P}$ be the set of all paths. In this paper, we study completeness-resolvable graphs and obtain the following result.

Theorem 1.1 Let $G$ be a connected graph. Then $G$ is completeness-resolvable if and only if $G$ is isomorphic to a graph in $\mathcal{P} \cup \mathcal{K} \cup \mathcal{B} \cup \mathcal{C}$, where $\mathcal{B}$ and $\mathcal{C}$ are as refer to Constructions 2.1 and 2.3, respectively.

The rest of this paper is organized as follows.

In Section 2, we first give some notions and notations, and then construct families $\mathcal{B}_{k}$ and $\mathcal{C}_{k}$ of graphs for each $k \geq 2$. The constructions depends on edge coverings of some vertices in given bipartite graphs. Actually, the sets $\mathcal{B}$ and $\mathcal{C}$ in Theorem 1.1 are equal to $\bigcup_{k=2}^{+\infty} \mathcal{B}_{k}$ and $\bigcup_{k=2}^{+\infty} \mathcal{C}_{k}$, respectively.

In Section 3, Theorem 1.1 is proved.

In Section 4, using the spanning subgraph relationship, we establish respective posets on $\mathcal{B}_{k}$ and $\mathcal{C}_{k}$. Based on each poset, we find the maximum graph and give the lower and upper bounds for the size of a minimal graph. Furthermore, we characterize the minimal graphs satisfying the lower or upper bound.

In Section 5, we first obtain the respective ranges for diameters of graphs in $\mathcal{B}_{k}$ and $\mathcal{C}_{k}$. Then we introduce the concept of perfectness-resolvable graphs, which are closely related to completeness-resolvable graphs. Finally, we give sufficient conditions to determine a perfectness-resolvable graph, and conclude this paper by raising a problem which graphs are perfectness-resolvable. 


\section{Constructions}

We first give some notions and notations that will be used throughout of this paper. Let $G$ be a graph. For $x \in V(G)$ and $e \in E(G)$, we say that e covers $x$, or $x$ is covered by $e$, if $e$ is incident to $x$ in $G$. For $S \subseteq V(G)$, an edge covering of $S$, or $S$-covering, is a family $E$ of edges in $G$ such that each vertex in $S$ is covered by at least one edge in $E$.

Notation 1 Given a graph $G$ and a subset $S \subseteq V(G)$, denote by $\mathcal{E}(G, S)$ the set of all $S$-coverings in $G$.

Notation 2 Let $k$ and $m$ be positive integers.

(i) For any vector $x \in[m]^{k}$, denote by $x_{(i)}$ the $i$ th component of $x$.

(ii) For $I \subseteq[k]$ and $J \subseteq[m]$, write

$$
[m]_{I}^{k}(J)=\left\{x \in[m]^{k} \mid x_{(i)} \in J \text { for all } i \in I\right\} .
$$

For simplify, we write $[m]_{i}^{k}(J),[m]_{I}^{k}(j)$ and $[m]_{i}^{k}(j)$ instead of $[m]_{\{i\}}^{k}(J),[m]_{I}^{k}(\{j\})$ and $[m]_{\{i\}}^{k}(\{j\})$, respectively.

(iii) For $S \subseteq[k] \cup[m]^{k}$, let $K_{S}$ and $\bar{K}_{S}$ denote the complete and null graphs on $S$, respectively.

(iv) Denote by $\mathcal{G}([k])$ and $\mathcal{G}\left([m]^{k}\right)$ the sets of all graphs with the vertex sets $[k]$ and $[m]^{k}$, respectively.

Let $G$ be a graph. A graph $H$ is a subgraph of $G$ if $V(H) \subseteq V(G)$ and $E(H) \subseteq$ $E(G)$. Furthermore, the subgraph $H$ is a spanning subgraph of $G$ if $V(H)=V(G)$. For $S \subseteq V(G)$, the induced subgraph of $G$ on $S$ is the graph with the vertex set $S$ such that two vertices are adjacent if and only if they are adjacent in $G$.

Notation 3 Let $k$ and $m$ be positive integers. For $E \subseteq E\left(K_{[m]^{k}}\right)$, denote by $\operatorname{span}(E)$ the spanning subgraph of $K_{[m]^{k}}$ with the edge set $E$.

Notation 4 Let $k$ and $m$ be positive integers. For $H_{1} \in \mathcal{G}([k])$ and $H_{2} \in \mathcal{G}\left([m]^{k}\right)$, define $H_{1} \circ H_{2}$ as the graph on the disjoined union $[k] \sqcup[m]^{k}$ with the edge set $E\left(H_{1}\right) \cup E\left(H_{2}\right) \cup E\left(H_{1}, H_{2}\right)$, where

$$
E\left(H_{1}, H_{2}\right)=\left\{\{i, x\} \mid i \in[k], x \in[m]^{k}, x_{(i)}=1\right\} .
$$

Remark 1 For $H_{1} \in \mathcal{G}([k])$ and $H_{2} \in \mathcal{G}\left([m]^{k}\right)$, we have

$$
\left|E\left(H_{1} \circ H_{2}\right)\right|=\left|E\left(H_{1}\right)\right|+\left|E\left(H_{2}\right)\right|+k \cdot m^{k-1} .
$$

Next, we construct a family $\mathcal{B}$ of graphs.

Construction 2.1 Let $\mathcal{B}=\bigcup_{k=2}^{+\infty} \mathcal{B}_{k}$, where $\mathcal{B}_{k}$ is defined by the following steps.

(i) For each $i \in[k]$, denote by $B_{i}^{k}$ the complete bipartite graph with two parts $[2]_{i}^{k}(1)$ and $[2]_{i}^{k}(2)$. 
(ii) Define

$$
\mathcal{B}_{k}=\left\{H_{1} \circ \operatorname{span}\left(\bigcup_{i \in[k]} E_{i}\right) \mid H_{1} \in \mathcal{G}([k]), E_{i} \in \mathcal{E}\left(B_{i}^{k},[2]_{H_{1}(i)}^{k}(2)\right)\right\},
$$

where $H_{1}(i)$ is the union of $\{i\}$ and the set of vertices adjacent to $i$ in $H_{1}$.

Observing that $\bigcup_{i \in[k]} E\left(B_{i}^{k}\right)=E\left(K_{[2]^{m}}\right)$, we have the following result immediately from Construction 2.1.

Proposition 2.2 For $k \geq 2$, pick $H_{1} \in \mathcal{G}([k])$ and $H_{2} \in \mathcal{G}\left([2]^{k}\right)$. Then $H_{1} \circ H_{2} \in \mathcal{B}_{k}$ if and only if the edge subset $L_{i}\left(H_{2}\right)$ is a $[2]_{H_{1}(i)}^{k}(2)$-covering for each $i \in[k]$, where

$$
L_{i}\left(H_{2}\right)=E\left(H_{2}\right) \cap E\left(B_{i}^{k}\right) .
$$

We now construct another family $\mathcal{C}$ of graphs.

Construction 2.3 Let $\mathcal{C}=\bigcup_{k=2}^{+\infty} \mathcal{C}_{k}$, where $\mathcal{C}_{k}$ is constructed by the following steps.

(i) For $i \in[k]$, let $C_{i}^{k}$ be the bipartite graph with two parts $[3]_{i}^{k}(1)$ and $[3]_{i}^{k}(2)$, where the edge set is

$$
\left\{\{x, y\}\left|x \in[3]_{i}^{k}(1), y \in[3]_{i}^{k}(2),\right| x_{(t)}-y_{(t)} \mid \leq 1 \text { for each } t \in[k] \backslash\{i\}\right\} .
$$

(ii) For $i \in[k]$, let $D_{i}^{k}$ be the bipartite graph with two parts $[3]_{i}^{k}(2)$ and $[3]_{i}^{k}(3)$, where the edge set is

$$
\left\{\{x, y\}\left|x \in[3]_{i}^{k}(2), y \in[3]_{i}^{k}(3),\right| x_{(t)}-y_{(t)} \mid \leq 1 \text { for each } t \in[k] \backslash\{i\}\right\} .
$$

(iii) Define

$$
\mathcal{C}_{k}=\left\{\bar{K}_{[k]} \circ \operatorname{span}\left(\bigcup_{i \in[k]}\left(E_{i} \cup F_{i}\right)\right) \mid E_{i} \in \mathcal{E}\left(C_{i}^{k},[3]_{i}^{k}(2)\right), F_{i} \in \mathcal{E}\left(D_{i}^{k}, S_{i}^{k}\right)\right\},
$$

where $S_{i}^{k}=[3]_{[k]}^{k}(\{2,3\}) \cap[3]_{i}^{k}(3)$.

We get the following result immediately from the construction of $\mathcal{C}_{k}$.

Proposition 2.4 For $k \geq 2$, choose $H_{2} \in \mathcal{G}\left([3]^{k}\right)$. Then $\bar{K}_{[k]} \circ H_{2} \in \mathcal{C}_{k}$ if and only if the following conditions hold.

(i) $E\left(H_{2}\right) \subseteq \bigcup_{i \in[k]}\left(E\left(C_{i}^{k}\right) \cup E\left(D_{i}^{k}\right)\right)$.

(ii) For each $i \in[k]$, the edge subset $M_{i}\left(H_{2}\right)$ is a $[3]_{i}^{k}(2)$-covering, where

$$
M_{i}\left(H_{2}\right)=E\left(H_{2}\right) \cap E\left(C_{i}^{k}\right) .
$$

(iii) For each $i \in[k]$, the edge subset $N_{i}\left(H_{2}\right)$ is an $S_{i}^{k}$-covering, where

$$
N_{i}\left(H_{2}\right)=E\left(H_{2}\right) \cap E\left(D_{i}^{k}\right) .
$$




\section{Proof of Theorem 1.1}

In this section, we always suppose that $G$ is a connected graph. If $W$ is a completenessresolving set of $G$ with $|W|=k$ and $m(W)=m$, we say that $W$ is a $(k, m)$ completeness-resolving set, or $(k, m)$-CRS for simplify, and $G$ is a $(k, m)$-completenessresolvable graph, or $(k, m)-C R G$ for simplify. The proof of Theorem 1.1 is divided in three subsections.

\section{$3.1 \quad k \geq 2$ and $m=2$}

In this subsection, we determine the set of all $(k, 2)$-CRGs for $k \geq 2$.

Lemma 3.1 With references to Construction 2.1, let $H$ be a graph in $\mathcal{B}_{k}$.

(i) For $i \in[k]$ and $x \in[2]^{k}$, we have $d(i, x)=x_{(i)}$.

(ii) The graph $H$ is a $(k, 2)-C R G$.

Proof. (i) Write $H=H_{1} \circ H_{2}$, where $H_{1} \in \mathcal{G}([k])$ and $H_{2} \in \mathcal{G}\left([2]^{k}\right)$. If $x_{(i)}=1$, then $d(i, x)=1=x_{(i)}$. Now suppose $x_{(i)}=2$. On one hand, since $i$ and $x$ are not adjacent in $H$, we have $d(i, x) \geq 2$. On the other hand, if $x \in[2]_{H_{1}(i)}^{k}(2)$, by Proposition 2.2, there is an edge $\{x, y\} \in L_{i}\left(H_{2}\right)$, then $y_{(i)}=1$, and so we get a path $(i, y, x)$ in $H$, which implies that $d(i, x) \leq 2$. If $x \notin[2]_{H_{1}(i)}^{k}(2)$, then there exists a vertex $t \in H_{1}(i) \backslash\{i\}$ such that $x_{(t)}=1$, and so we get a path $(i, t, x)$ in $H$, which implies that $d(i, x) \leq 2$. Consequently, one has $d(i, x)=2=x_{(i)}$.

(ii) It follows from (i) that $\Phi_{[k]}(x)=x$ for each $x \in[2]^{k}$. Therefore, we have derived that $\Phi_{[k]}$ is a bijection, which implies that $[k]$ is a $(k, 2)$-CRS, and so $H$ is a $(k, 2)-\mathrm{CRG}$, as desired.

Proposition 3.2 For $k \geq 2$, a graph $G$ is a $(k, 2)-C R G$ if and only if $G$ is isomorphic to a graph in $\mathcal{B}_{k}$.

Proof. The sufficiency holds by Lemma 3.1 (ii). To prove the necessity, suppose that $G$ is a $(k, 2)$-CRG and let $W=\left\{w_{1}, \ldots, w_{k}\right\}$ be a $(k, 2)$-CRS of $G$. Then

$$
\Psi_{W}: V(G) \backslash W \longrightarrow[2]^{k}, \quad u \longmapsto\left(d\left(w_{1}, u\right), \ldots, d\left(w_{k}, u\right)\right)
$$

is a bijection. Define a graph $H$ on the set $[k] \cup[2]^{k}$ with the edge set $E_{[k]} \cup E_{[2]^{k}} \cup$ $E_{[k],[2]}$, where

$$
\begin{aligned}
E_{[k]} & =\left\{\{i, j\} \mid i, j \in[k],\left\{w_{i}, w_{j}\right\} \in E(G)\right\}, \\
E_{[2]^{k}} & =\left\{\{x, y\} \mid x, y \in[2]^{k},\left\{\Psi_{W}^{-1}(x), \Psi_{W}^{-1}(y)\right\} \in E(G)\right\}, \\
E_{[k],[2]^{k}} & =\left\{\{i, x\} \mid i \in[k], x \in[2]^{k}, x_{(i)}=1\right\} .
\end{aligned}
$$

Note that $x_{(i)}=d\left(w_{i}, \Psi_{W}^{-1}(x)\right)$. It is routine to verify that

$$
\Psi: V(G) \longrightarrow[k] \cup[2]^{k}, \quad u \longmapsto \begin{cases}i, & \text { if } u=w_{i}, \\ \Psi_{W}(u), & \text { if } u \in V(G) \backslash W\end{cases}
$$


is an isomorphism from $G$ to $H$. Hence, graphs $G$ and $H$ are isomorphic. Now it suffices to prove $H \in \mathcal{B}_{k}$.

Let $H_{1}$ and $H_{2}$ be the induced subgraphs of $H$ on $[k]$ and $[2]^{k}$, respectively. Then $H=H_{1} \circ H_{2}$ by (5). Let $i \in[k]$. Pick any vertex $x \in[2]_{H_{1}(i)}^{k}(2)$. For each $t \in H_{1}(i)$, we have $d\left(w_{t}, \Psi_{W}^{-1}(x)\right)=x_{(t)}=2$. Particularly, one gets $d\left(w_{i}, \Psi_{W}^{-1}(x)\right)=2$. Hence, there is $u \in V(G) \backslash W$ such that $\left(w_{i}, u, \Psi_{W}^{-1}(x)\right)$ is a path in $G$, which implies that $\left(i, \Psi_{W}(u), x\right)$ is a path in $H$, and so $\left\{\Psi_{W}(u), x\right\} \in L_{i}\left(H_{2}\right)$, where $L_{i}\left(H_{2}\right)$ is as refer to (2). From the arbitrary choice of $x$ in $[2]_{H_{1}(i)}^{k}(2)$, we have $L_{i}\left(H_{2}\right) \in \mathcal{E}\left(B_{i}^{k},[2]_{H_{1}(i)}^{k}(2)\right)$. It follows from Proposition 2.2 that $H \in \mathcal{B}_{k}$, as desired.

\section{$3.2 \quad k \geq 2$ and $m=3$}

In this subsection, we determine the set of all $(k, 3)$-CRGs for $k \geq 2$.

Lemma 3.3 With references to Construction 2.3, let $H$ be a graph in $\mathcal{C}_{k}$.

(i) For $i \in[k]$ and $x \in[3]^{k}$, we have $d(i, x)=x_{(i)}$.

(ii) The graph $H$ is a $(k, 3)-C R G$.

Proof. (i) Write $H=\bar{K}_{[k]} \circ H_{2}$, where $H_{2} \in \mathcal{G}\left([3]^{k}\right)$. Note that $x_{(i)} \in\{1,2,3\}$.

Case 1. $x_{(i)}=1$. Then $d(i, x)=1=x_{(i)}$.

Case 2. $x_{(i)}=2$. Since $x \in[3]_{i}^{k}(2)$, from Proposition 2.4 (ii), there is an edge $\{x, y\} \in M_{i}\left(H_{2}\right)$ for $y \in[3]_{i}^{k}(1)$, and so we obtain a path $(i, y, x)$ in $H$, which implies that $d(i, x)=2=x_{(i)}$.

Case 3. $x_{i}=3$. Then $x \in[3]_{i}^{k}(3)$. On one hand, for any $x^{\prime} \in[3]_{i}^{k}(1)$, since $\left|x_{(i)}-x_{(i)}^{\prime}\right|=2>1$, according to Proposition 2.4 (i), vertices $x$ and $x^{\prime}$ are not adjacent in $H_{2}$, which implies that $d\left(x^{\prime}, x\right) \geq 2$. Hence, we get $d(i, x) \geq 3$. On the other hand, if $x \in S_{i}^{k}$, then by Proposition 2.4 (iii), there is an edge $\{x, z\} \in N_{i}\left(H_{2}\right)$ for $z \in[3]_{i}^{k}(2)$, and further by Proposition 2.4 (ii), there is an edge $\{z, v\} \in M_{i}\left(H_{2}\right)$ for $v \in[3]_{i}^{k}(1)$, which implies that there is a path $(i, v, z, x)$ in $H$, and so $d(i, x) \leq 3$; If $x \notin S_{i}^{k}$, then there is $t \in[k]$ with $x_{(t)}=1$, which indicates that $(i,(1, \ldots, 1), t, x)$ is a path in $H$, and so $d(i, x) \leq 3$. Consequently, we get $d(i, x)=3=x_{(i)}$.

Combining all these three cases, we obtain (i).

(ii) It follows from (i) that $\Phi_{[k]}(x)=x$ for each $x \in[3]^{k}$. This indicates that $\Phi_{[k]}$ is a bijection, and so $H$ is a $(k, 3)-C R G$, as desired.

Lemma 3.4 Let $W$ be a $(k, 3)-C R S$ of a graph $G$.

(i) Then the induced subgraph of $G$ on $W$ is a null graph.

(ii) For $u, v \in V(G) \backslash W$, write $x=\Phi_{W}(u)$ and $y=\Phi_{W}(v)$. If $u$ and $v$ are adjacent in $G$, then $\left|x_{(i)}-y_{(i)}\right| \leq 1$ for each $i \in[k]$.

Proof. Let $W=\left\{w_{1}, w_{2}, \ldots, w_{k}\right\}$.

(i) Suppose for the contrary that there are vertices $w_{i}, w_{j} \in W$ such that $\left\{w_{i}, w_{j}\right\}$ is an edge in $G$. Without loss of generality, assume that $\left\{w_{i}, w_{j}\right\}=\left\{w_{1}, w_{2}\right\}$. Since $\Phi_{W}$ is an bijection, there is a vertex $u_{0} \in V(G) \backslash W$ such that $\Phi_{W}\left(u_{0}\right)=(3,1, \ldots, 1)$, which implies that

$$
3=d\left(w_{1}, u_{0}\right) \leq d\left(w_{1}, w_{2}\right)+d\left(w_{2}, u_{0}\right)=1+1,
$$


a contradiction.

(ii) By contradiction, suppose that there exists $i \in[k]$ such that $\left|x_{(i)}-y_{(i)}\right| \geq 2$. Noting that $x, y \in[3]^{k}$, we have $\left\{x_{(i)}, y_{(i)}\right\}=\{1,3\}$. Without loss of generality, assume that $x_{(i)}=1$ and $y_{(i)}=3$. Then

$$
3=y_{(i)}=d\left(w_{i}, v\right) \leq d\left(w_{i}, u\right)+d(u, v)=x_{(i)}+1=2,
$$

a contradiction.

Proposition 3.5 For $k \geq 2$, a graph $G$ is a $(k, 3)-C R G$ if and only if $G$ is isomorphic to a graph in $\mathcal{C}_{k}$.

Proof. The sufficiency holds by Lemma 3.3 (ii). Substituting $[3]^{k}$ for $[2]^{k}$ in the proof of Proposition 3.2, we can obtain a graph $H=H_{1} \circ H_{2}$ and an isomorphism $\Phi$ from $G$ to $H$, where $H_{1}$ and $H_{2}$ are graphs defined on the set $[k]$ and $[3]^{k}$, respectively. To get the necessity, it is enough to prove $H \in \mathcal{C}_{k}$.

Noting that $\Phi^{-1}([k])$ is a $(k, 3)$-CRS of $G$, we infer from Lemma 3.4 (i) that $H_{1}=\bar{K}_{[k]}$. Combining Lemma 3.4 (ii) and Proposition 2.4, we only need to show that $M_{i}\left(H_{2}\right) \in \mathcal{E}\left(C_{i}^{k},[3]_{i}^{k}(2)\right)$ and $N_{i}\left(H_{2}\right) \in \mathcal{E}\left(D_{i}^{k}, S_{i}^{k}\right)$ for each $i \in[k]$.

Pick any vertex $x \in[3]_{i}^{k}(2)$. Then $d(i, x)=x_{(i)}=2$ by Lemma 3.3 (i). Since $H_{1}$ is null, there is a vertex $x^{\prime} \in[3]^{k}$ such that $\left(i, x^{\prime}, x\right)$ is a path in $H$, which implies that $x_{(i)}^{\prime}=1$, and so $\left\{x^{\prime}, x\right\} \in M_{i}\left(H_{2}\right)$ by Lemma 3.4 (ii). From the arbitrary choice of $x$ in $[3]_{i}^{k}(2)$, we have $M_{i}\left(H_{2}\right) \in \mathcal{E}\left(C_{i}^{k},[3]_{i}^{k}(2)\right)$.

Pick any vertex $y \in S_{i}^{k}$. Then $d(i, y)=y_{(i)}=3$ by Lemma 3.3 (i). Hence, there exist vertices $y^{\prime}, y^{\prime \prime} \in[k] \cup[3]^{k}$ such that $\left(i, y^{\prime \prime}, y^{\prime}, y\right)$ is a path in $H$. Since $d(t, y)=y_{(t)} \geq 2$ for each $t \in[k]$, one gets $y^{\prime} \in[3]^{k}$. Noting that $H_{1}=\bar{K}_{[k]}$, we have $y^{\prime \prime} \in[3]^{k}$ and furthermore $y_{(i)}^{\prime \prime}=1$. It follows from Lemma 3.4 (ii) that $\left|y_{(i)}^{\prime \prime}-y_{(i)}^{\prime}\right| \leq 1$ and $\left|y_{(i)}^{\prime}-y_{(i)}\right| \leq 1$, and so $y_{(i)}^{\prime}=2$, which implies that $\left\{y^{\prime}, y\right\} \in N_{i}\left(H_{2}\right)$. By the arbitrary choice of $y$ in $S_{i}^{k}$, we have $N_{i}\left(H_{2}\right) \in \mathcal{E}\left(D_{i}^{k}, S_{i}^{k}\right)$.

The proof is completed.

\subsection{Proof of Theorem 1.1}

Proposition 3.6 A graph $G$ is a $(1, m)-C R G$ if and only if $G$ is a path.

Proof. If $G$ is a $(1, m)$-CRG, then $G$ has a resolving set of cardinality 1 , and so $G$ is a path. Conversely, if $G$ is a path $\left(u_{0}, u_{1}, \ldots, u_{m}\right)$, then $\Phi_{\left\{u_{0}\right\}}\left(u_{i}\right)=(i)$ for $i \in[m]$, which implies that $\left\{u_{0}\right\}$ is a $(1, m)$-CRS, and so $G$ is a $(1, m)-\mathrm{CRG}$, as desired.

Proposition 3.7 A graph $G$ is a $(k, 1)-C R G$ if and only if the order of $G$ is $k+1$ and $G$ has a universal vertex.

Proof. Note that a vertex $u$ of $G$ is universal if and only if

$$
\Phi_{V(G) \backslash\{u\}}(u)=(1, \ldots, 1) .
$$

Hence, the desired result follows. 
Lemma 3.8 If $G$ is $a(k, m)-C R G$ with $k \geq 2$ and $m \geq 2$, then $m=2$ or 3 .

Proof. By contradiction, suppose $m \geq 4$. Let $W=\left\{w_{1}, w_{2}, \ldots, w_{k}\right\}$ be a $(k, m)$ CRS of $G$. Since $\Phi_{W}$ is a bijection, there exist vertices $u$ and $v$ in $V(G) \backslash W$ such that $\Phi_{W}(u)=(1,1, \ldots, 1)$ and $\Phi_{W}(v)=(4,1, \ldots, 1)$, which implies that

$$
4=d\left(w_{1}, v\right) \leq d\left(w_{1}, u\right)+d\left(u, w_{2}\right)+d\left(w_{2}, v\right)=1+1+1,
$$

a contradiction.

Theorem 1.1 follows from Lemma 3.8 and Propositions 3.2, 3.5, 3.6 and 3.7.

\section{Partially ordered sets}

In Theorem 1.1, graphs in $\mathcal{P} \cup \mathcal{K}$ are well-understood, however, graphs in $\mathcal{B} \cup \mathcal{C}$ are non-intuitive. Now the letter $k$ is always used to denote a given positive integer at least 2. Note that graphs in $\mathcal{B}_{k}$ (resp. $\mathcal{C}_{k}$ ) have the same vertex set $[2]^{k}$ (resp. $[3]^{k}$ ).

Notation 5 For graphs $G_{1}$ and $G_{2}$ with the same vertex set, define $G_{1} \preceq G_{2}$ if $G_{1}$ is a spanning subgraph of $G_{2}$. Write $G_{1} \prec G_{2}$ if $G_{1} \preceq G_{2}$ and $G_{1} \neq G_{2}$.

A partially ordered set, or poset, is an ordered pair $(A, \leq)$ such that $\leq$ is a reflexive, antisymmetric and transitive binary relation on the set $A$. An element $x$ is maximum in $A$ if $a \leq x$ for each $a \in A$. An element $y$ is minimal in $A$ if $a \leq y$ implies $a=y$. Observe that $\left(\mathcal{B}_{k}, \preceq\right)$ and $\left(\mathcal{C}_{k}, \preceq\right)$ are posets on graphs.

In Subsection 4.1 (resp. 4.2$)$, based on the poset $\left(\mathcal{B}_{k}, \preceq\right)\left(\operatorname{resp} .\left(\mathcal{C}_{k}, \preceq\right)\right.$ ), we first obtain the maximum graph in $\mathcal{B}_{k}$ (resp. $\mathcal{C}_{k}$ ), and turn the problem of characterizing graphs in $\mathcal{B}_{k}$ (resp. $\mathcal{C}_{k}$ ) into characterizing minimal graphs in $\mathcal{B}_{k}$ (resp. $\mathcal{C}_{k}$ ). Then we investigate minimal graphs in $\mathcal{B}_{k}\left(\right.$ resp. $\left.\mathcal{C}_{k}\right)$.

In Subsection 4.1, noting from Construction 2.1 that the first factor of a graph in $\mathcal{B}_{k}$ is an arbitrary graph in $\mathcal{G}([k])$, we fix $H_{1} \in \mathcal{G}([k])$ and give a definition of an $H_{1}$-minimal graph. Then the size of an $H_{1}$-minimal graph is bounded by using the degrees of vertices in $H_{1}$, and furthermore, we describe the $H_{1}$-minimal graphs satisfying the lower or upper bound. As examples, we apply the results to study $K_{[k]}$-minimal graphs and $\bar{K}_{[k]}$-minimal graphs.

In Subsection 4.2, noting from Construction 2.3 that the first factor of a graph in $\mathcal{C}_{k}$ is the null graph in $\mathcal{G}([k])$, we say that $H_{2}$ is $k$-minimal if $\bar{K}_{[k]} \circ H_{2}$ is a minimal graph in $\mathcal{C}_{k}$. We establish the lower and upper bounds for the size of a $k$-minimal graph, and characterize all $k$-minimal graphs satisfying the lower or upper bound.

\subsection{A poset on $\mathcal{B}_{k}$}

In this subsection, we focus on the poset $\left(\mathcal{B}_{k}, \preceq\right)$, and always suppose that $H_{1} \in$ $\mathcal{G}([k])$ and $H_{2} \in \mathcal{G}\left([2]^{k}\right)$.

Theorem 4.1 Suppose that $H_{1} \circ H_{2} \in \mathcal{B}_{k}$. If $H_{1} \preceq H_{1}^{\prime} \preceq K_{[k]}$ and $H_{2} \preceq H_{2}^{\prime} \preceq K_{[2]^{k}}$, then $H_{1}^{\prime} \circ H_{2}^{\prime} \in \mathcal{B}_{k}$. 
Proof. For each $i \in[k]$, we get $[2]_{H_{1}^{\prime}(i)}^{k}(2) \subseteq[2]_{H_{1}(i)}^{k}(2)$ and $L_{i}\left(H_{2}\right) \subseteq L_{i}\left(H_{2}^{\prime}\right)$, where $L_{i}\left(H_{2}\right)$ is as refer to (2). Hence, the desired result follows by Proposition 2.2.

Let $G_{1}$ and $G_{2}$ be graphs with the same vertex set $V$. The union $G_{1} \cup G_{2}$ is the graph with the vertex set $V$ and the edge set $E\left(G_{1}\right) \cup E\left(G_{2}\right)$.

Corollary 4.2 For $G_{1}, G_{2} \in \mathcal{B}_{k}$, we have $G_{1} \cup G_{2} \in \mathcal{B}_{k}$. Particularly, we have

$$
\bigcup_{G \in \mathcal{B}_{k}} G=K_{[k]} \circ K_{[2]^{k}} \in \mathcal{B}_{k} .
$$

Proof. Let $G_{1}=H_{1} \circ H_{2}$ and $G_{2}=H_{1}^{\prime} \circ H_{2}^{\prime}$. Then $G_{1} \cup G_{2}=\left(H_{1} \cup H_{1}^{\prime}\right) \circ\left(H_{2} \cup H_{2}^{\prime}\right)$. Hence, the desired result follows from Theorem 4.1.

A join-semilattice is a poset in which any pair of elements has the least upper bound. Note that each finite join-semilattice has a unique maximum element. The following result is immediate from Corollary 4.2.

Proposition 4.3 The poset $\left(\mathcal{B}_{k}, \preceq\right)$ is a finite join-semilattice with the maximum graph $K_{[k]} \circ K_{[2] k}$.

From Theorem 4.1, it is nature to put forward the following notion. We say that $H_{2}$ is $H_{1}$-minimal if $H_{1} \circ H_{2} \in \mathcal{B}_{k}$ and $H_{1} \circ H_{2}^{\prime} \notin \mathcal{B}_{k}$ for $H_{2}^{\prime} \prec H_{2}$.

Remark 2 If $H_{1} \circ H_{2}$ is a minimal graph in $\mathcal{B}_{k}$, then $H_{2}$ is $H_{1}$-minimal. However, the converse is not true.

We get the following result from Proposition 2.2.

Lemma 4.4 $\mathrm{A}$ graph $\mathrm{H}_{2}$ is $\mathrm{H}_{1}$-minimal if and only if the following conditions hold.

(i) For each $i \in[k]$, the edge subset $L_{i}\left(H_{2}\right)$, as refer to $(2)$, is a $[2]_{H_{1}(i)}^{k}$-covering.

(ii) For each $e \in E\left(H_{2}\right)$, there exists a vertex $i \in[k]$ such that $L_{i}\left(H_{2}\right) \backslash\{e\}$ is not a $[2]_{H_{1}(i)}^{k}$-covering.

Notation 6 Let $H_{1} \in \mathcal{G}([k])$ and $H_{2} \in \mathcal{G}\left([2]^{k}\right)$.

(i) For $x \in[2]^{k}$, let $J_{x}=\left\{i \in[k] \mid x \in[2]_{H_{1}(i)}^{k}(2)\right\}$.

(ii) For $x \in[2]^{k}$ and $e \in E\left(H_{2}\right)$, write

$$
I_{x}(e)=\left\{i \in[k] \mid x \in[2]_{H_{1}(i)}^{k}(2), e \in L_{i}\left(H_{2}\right) \text {, e covers } x\right\},
$$

and furthermore, let

$$
I_{x}=\bigcup_{f \in E\left(H_{2}\right)} I_{x}(f) \quad \text { and } \quad \tilde{I}_{x}(e)=I_{x} \backslash\left(\bigcup_{f \in E\left(H_{2}\right) \backslash\{e\}} I_{x}(f)\right) .
$$

Lemma 4.5 A graph $\mathrm{H}_{2}$ is $\mathrm{H}_{1}$-minimal if and only if the following conditions hold.

(i) For each $x \in[2]^{k}$, we have $I_{x}=J_{x}$.

(ii) For each $e \in E\left(H_{2}\right)$, there is a vertex $x \in[2]^{k}$ such that $\tilde{I}_{x}(e) \neq \emptyset$. 
Proof. Note that $I_{x} \subseteq J_{x}$. Observing that $i \in J_{x}$ if and only if $x \in[2]_{H_{1}(i)}^{k}(2)$, we obtain (i) if and only if the condition (i) in Lemma 4.4 holds.

Now suppose that the condition (i) in Lemma 4.4 holds. Then $i \in \tilde{I}_{x}(e)$ if and only if any edge in $L_{i}\left(H_{2}\right) \backslash\{e\}$ does not cover $x$. Hence, we obtain (ii) if and only if the condition (ii) in Lemma 4.4 holds.

Consequently, the desired result follows from Lemma 4.4.

Actually, given a graph $H_{1}$, the $H_{1}$-minimal graph $H_{2}$ is not unique. We give lower and upper bounds for the size of $\mathrm{H}_{2}$. The degree of a vertex in a graph is the number of edges covering this vertex in the graph.

Theorem 4.6 If $\mathrm{H}_{2}$ is $\mathrm{H}_{1}$-minimal, then

$$
2^{k-\min \left\{d_{i} \mid i \in[k]\right\}-1} \leq\left|E\left(H_{2}\right)\right| \leq \sum_{i=1}^{k} 2^{k-d_{i}-1},
$$

where $d_{i}$ is the degree of the vertex $i$ in $H_{1}$.

Proof. For each $i \in[k]$, write $E_{i}=L_{i}\left(H_{2}\right)$ and denote by $E_{i}^{\prime}$ the set of edges $e$ such that $L_{i}\left(H_{2}\right) \backslash\{e\}$ is not a $[2]_{H_{1}(i)}^{k}$-covering. By Lemma 4.4 (ii), we have

$$
\max \left\{\left|E_{i}\right| \mid i \in[k]\right\} \leq\left|\bigcup_{i \in[k]} E_{i}\right|=\left|E\left(H_{2}\right)\right|=\left|\bigcup_{i \in[k]} E_{i}^{\prime}\right| \leq \sum_{i \in[k]}\left|E_{i}^{\prime}\right| .
$$

Noting that $\left|E_{i}^{\prime}\right| \leq 2^{k-\left|H_{1}(i)\right|}=2^{k-d_{i}-1} \leq\left|E_{i}\right|$, we get the desired result.

Remark 3 If $H_{1} \circ H_{2}$ is a minimal graph in $\mathcal{B}_{k}$, then combining the equation (1), Remark 2 and Theorem 4.6, we get

$k \cdot 2^{k-1}+\frac{1}{2} \sum_{i=1}^{k} d_{i}+2^{k-\min \left\{d_{i} \mid i \in[k]\right\}-1} \leq\left|E\left(H_{1} \circ H_{2}\right)\right| \leq k \cdot 2^{k-1}+\frac{1}{2} \sum_{i=1}^{k}\left(2^{k-d_{i}}+d_{i}\right)$.

The minimum degree of a graph is the minimum value of degrees of all vertices in this graph. We use the following result to characterize the bounds in Theorem 4.6.

Corollary 4.7 Suppose that $H_{2}$ is $H_{1}$-minimal.

(i) The lower bound in Theorem 4.6 is attained if and only if there exists a vertex $i$ with minimum degree in $H_{1}$ such that $L_{i}\left(H_{2}\right)=E\left(H_{2}\right)$.

(ii) The upper bound in Theorem 4.6 is attained if and only if for any distinct vertices $x, y \in[2]^{k}$ and any distinct edges e, $f \in E\left(H_{2}\right)$, we have (a) $\left|I_{x}(e)\right| \leq 1$, (b) either $I_{x}(e)=\emptyset$ or $I_{y}(e)=\emptyset$, (c) $I_{x}(e) \cap I_{x}(f)=\emptyset$.

Proof. (i) It is immediate from the proof of the lower bound in Theorem 4.6.

(ii) Suppose that (a), (b) and (c) hold. Observing that $\tilde{I}_{x}(e) \subseteq I_{x}(e)$, we infer from Lemma 4.5 (ii) that

$$
\begin{aligned}
E\left(H_{2}\right) & =\bigcup_{x \in[2]^{k}}\left\{e \in E\left(H_{2}\right) \mid \tilde{I}_{x}(e) \neq \emptyset\right\}=\bigcup_{i \in[k]} \bigcup_{x \in[2]_{H_{1}(i)}^{k}(2)}\left\{e \in E\left(H_{2}\right) \mid i \in I_{x}(e)\right\} \\
& =\bigcup_{i \in[k]}\left\{e \in E\left(H_{2}\right) \mid i \in I_{x}(e) \text { for a certain vertex } x \in[2]_{H_{1}(i)}^{k}(2)\right\},
\end{aligned}
$$


and so $\left|E\left(H_{2}\right)\right|=\sum_{i \in[k]}\left|[2]_{H_{1}(i)}^{k}(2)\right|$, attaining the upper bound in Theorem 4.6.

In the following, suppose that $\left|E\left(H_{2}\right)\right|$ attains the upper bound in Theorem 4.6. With references to the proof of Theorem 4.6, the following conditions hold.

(C1) $\left|E_{i}^{\prime}\right|=2^{k-d_{i}-1}$ for each $i \in[k]$.

(C2) $E_{i}^{\prime} \cap E_{j}^{\prime}=\emptyset$ for any distinct vertices $i$ and $j$ in $[k]$.

By $(\mathrm{C} 1)$, the following condition holds for each $i \in[k]$.

$\left(\mathrm{C}^{\prime}\right)$ For each $x \in[2]_{H_{1}(i)}^{k}(2)$, there is a unique edge $e \in E_{i}^{\prime}$ such that $e$ covers $x$. Now we divide the proof in three steps.

We first prove (c). By contradiction, suppose $I_{x}(e) \cap I_{x}(f) \neq \emptyset$. Pick $i \in$ $I_{x}(e) \cap I_{x}(f)$. Then $x \in[2]_{H_{1}(i)}^{k}(2),\{e, f\} \subseteq L_{i}\left(H_{2}\right)$ and both $e$ and $f$ cover $x$, which implies that there is no edge in $E_{i}^{\prime}$ covering $x$, contrary to $\left(\mathrm{C}^{\prime}\right)$.

The next thing is to prove (a). Suppose for the contrary that $\left|I_{x}(e)\right| \geq 2$. Pick distinct vertices $i, j \in I_{x}(e)$. Note that $E_{i}^{\prime} \subseteq L_{i}\left(H_{2}\right)$ and $E_{j}^{\prime} \subseteq L_{j}\left(H_{2}\right)$. Combining $\left(\mathrm{C}^{\prime}\right)$ and (c), we get $e \in E_{i}^{\prime} \cap E_{j}^{\prime}$, contrary to (C2).

Finally, we prove (b). By contradiction, suppose that $I_{x}(e) \neq \emptyset$ and $I_{y}(e) \neq \emptyset$. Then $e=\{x, y\}$. Taking $i \in I_{x}(e)$ and $j \in I_{y}(e)$, we have $\left(y_{(i)}, x_{(i)}\right)=(1,2)$ and $\left(x_{(j)}, y_{(j)}\right)=(1,2)$, which implies that $i \neq j$. An argument similar to the one used in the previous step shows that $e \in E_{i}^{\prime} \cap E_{j}^{\prime}$, contrary to (C2).

The proof is complete.

As applications, we study $K_{[k]}$-minimal graphs and $\bar{K}_{[k]}$-minimal graphs in the rest of this subsection. To inverstigate $K_{[k]}$-minimal graphs, we observe that

$$
[2]_{K_{[k]}(i)}^{k}(2)=\{(2, \ldots, 2)\},
$$

and then get the following example from Lemma 4.5.

Example 4.8 (i) Define $\mathbb{U}_{k}$ as the graph with the vertex set $[2]^{k}$ and the edge set

$$
E\left(\mathbb{U}_{k}\right)=\{(1, \ldots, 1),(2, \ldots, 2)\} .
$$

Then $\mathbb{U}_{k}$ is a $K_{[k]}$-minimal graph with size 1 .

(ii) Define $\mathbb{V}_{k}$ as the graph with the vertex set $[2]^{k}$ and the edge set

$$
E\left(\mathbb{V}_{k}\right)=\bigcup_{i \in[k]}\left\{\{x,(2, \ldots, 2)\} \mid x_{(i)}=1, x_{(t)}=2 \text { for each } t \in[k] \backslash\{i\}\right\} .
$$

Then $\mathbb{V}_{k}$ is a $K_{[k]}$-minimal graph with size $k$.

Corollary 4.9 If $H_{2}$ is $K_{[k]}$-minimal, then $1 \leq\left|E\left(H_{2}\right)\right| \leq k$,

(i) with the lower bound if and only if $H_{2}=\mathbb{U}_{k}$.

(ii) with the upper bound if and only if $H_{2}=\mathbb{V}_{k}$.

Proof. Noting that the degree of each vertex $i$ in $K_{[k]}$ is $k-1$, we have $1 \leq\left|E\left(H_{2}\right)\right| \leq$ $k$ by Theorem 4.6.

(i) The "if" implication follows immediately from Example 4.8 (i), while the "only if" implication follows from (6) and Lemma 4.5 (i). 
(ii) We get the sufficiency from Example 4.8 (ii). To prove the necessity, suppose $\left|E\left(H_{2}\right)\right|=k$. Write $y=(2, \ldots, 2)$. By $(6)$, we have $J_{y}=[k]$ and $J_{z}=\emptyset$ for $z \in[2]^{k} \backslash\{y\}$. Noting that $\left|E\left(H_{2}\right)\right|$ attains the upper bound in Theorem 4.6, we deduced from Corollary 4.7 (ii) and Lemma 4.5 that $\left|I_{y}(e)\right|=1$ for $e \in E\left(H_{2}\right)$ and $\left\{I_{y}(e) \mid e \in E\left(H_{2}\right)\right\}$ is a partition of $[k]$. For any $e \in E\left(H_{2}\right)$, write $e=\{x, y\}$. Choosing the unique vertex $i \in I_{y}(e)$, we have $x_{(i)}=1$ and $x_{(t)}=2$ for each $t \in[k] \backslash\{i\}$, as desired.

To investigate $\bar{K}_{[k]}$-minimal graphs, we observe that

$$
[2] \frac{k}{K_{[k]}(i)}(2)=[2]_{i}^{k}(2),
$$

and for each $x \in[2]^{k}$, we have

$$
J_{x}=\left\{i \in[k] \mid x_{(i)}=2\right\} .
$$

Example 4.10 Define $\mathbb{R}_{k}$ as the graph with the vertex set $[2]^{k}$ and the edge set

$$
E\left(\mathbb{R}_{k}\right)=\left\{\{x, y\} \mid x, y \in[2]^{k}, x_{(i)} \neq y_{(i)} \text { for each } i \in[k]\right\} .
$$

Then $\mathbb{R}_{k}$ is a $\bar{K}_{[k]}$-minimal graph with size $2^{k-1}$.

Proof. Noting that $E\left(\mathbb{R}_{k}\right)$ is a matching, we infer that $\mathbb{R}_{k}$ has size $2^{k-1}$ and each vertex is covered by a unique edge in $\mathbb{R}_{k}$. Observing that $L_{i}\left(\mathbb{R}_{k}\right)=E\left(\mathbb{R}_{k}\right)$ for each $i \in[k]$, we conclude that $\mathbb{R}_{k}$ is $\bar{K}_{[k]}$-minimal from Lemma 4.4 and (7).

Remark 4 Actually, if $H_{1}$ has an isolated vertex, then $\mathbb{R}_{k}$ is $H_{1}$-minimal. However, if $\bar{K}_{[k]} \prec H_{1}$, then $H_{1} \circ \mathbb{R}_{k}$ is not a minimal graph in $\mathcal{B}_{k}$.

Given a graph $G$, the Cartesian product of $s$ copies of $G$ is the graph $G^{\square s}$ with the vertex set

$$
V\left(G^{\square s}\right)=\left\{\left(x_{(1)}, \ldots, x_{(s)}\right) \mid x_{(i)} \in V(G) \text { for } i \in[s]\right\},
$$

where two vertices $x$ and $y$ are adjacent if and only if there exists an index $i \in[s]$ such that $\left\{x_{(i)}, y_{(i)}\right\} \in E(G)$ and $x_{(j)}=y_{(j)}$ for all indices $j \in[s] \backslash\{i\}$.

Example 4.11 Let $P_{2}$ be the graph with the vertex set $\{1,2\}$ and the edge set $\{\{1,2\}\}$. Then $P_{2}^{\square k}$ is a $\bar{K}_{[k]}$-minimal graph with size $k \cdot 2^{k-1}$.

Proof. It is routine to verify that $\left|E\left(P_{2}^{\square k}\right)\right|=k \cdot 2^{k-1}$. For an edge $\{x, y\}$ in $P_{2}^{\square k}$ with $\left(x_{(i)}, y_{(i)}\right)=(1,2)$, by $(7)$, we have $I_{x}(\{x, y\})=\emptyset$ and $I_{y}(\{x, y\})=\{i\}$, and so $\tilde{I}_{y}(\{x, y\})=\{i\}$. By Lemma 4.5 and (8), we conclude that $P_{2}^{\square k}$ is $\bar{K}_{[k]}$-minimal.

Corollary 4.12 If $\mathrm{H}_{2}$ is $\bar{K}_{[k]}$-minimal, then $2^{k-1} \leq\left|E\left(H_{2}\right)\right| \leq k \cdot 2^{k-1}$,

(i) with the lower bound if and only if $H_{2}=\mathbb{R}_{k}$.

(ii) with the upper bound if and only if $\mathrm{H}_{2}=P_{2}^{\square k}$. 
Proof. Since the degree of each vertex $i$ in $\bar{K}_{[k]}$ is 0 , by Theorem 4.6, we have $2^{k-1} \leq\left|E\left(H_{2}\right)\right| \leq k \cdot 2^{k-1}$.

(i) The "if" implication follows immediately from Example 4.10, while the "only if" implication follows from (7) and Corollary 4.7 (i).

(ii) We get the sufficiency from Example 4.11. To prove the necessity, suppose that the size of $H_{2}$ is $k \cdot 2^{k-1}$, as well as the upper bound in Theorem 4.6 for $H_{1}=\bar{K}_{[k]}$. For $x \in[2]^{k}$, write $\varepsilon(x)=\left\{e \in E\left(H_{2}\right) \mid I_{x}(e) \neq \emptyset\right\}$. It follows from (8) and Lemma 4.5 (i) that $\varepsilon(x)=\emptyset$ if and only if $x=(1, \ldots, 1)$. Observing that $\tilde{I}_{x}(e) \subseteq I_{x}(e)$, we have derived from Lemma 4.5 (ii) that

$$
E\left(H_{2}\right)=\bigcup_{x \in[2]^{k}}\left\{e \in E\left(H_{2}\right) \mid I_{x}(e) \neq \emptyset\right\}=\bigcup_{x \in[2]^{k} \backslash\{(1, \ldots, 1)\}} \varepsilon(x) .
$$

Pick any vertex $x \in[2]^{k} \backslash\{(1, \ldots, 1)\}$. Combining Corollary 4.7 (ii) and Lemma 4.5 (i), we get $\left|I_{x}(e)\right|=1$ for any $e \in \varepsilon(x)$ and $\left\{I_{x}(e) \mid e \in \varepsilon(x)\right\}$ is a partition of $J_{x}$. For any edge $e=\{y, x\} \in \varepsilon(x)$, taking the unique vertex $i \in I_{x}(e)$, one has $\left(y_{(i)}, x_{(i)}\right)=(1,2)$ and $\left(y_{(t)}, x_{(t)}\right)=(2,2)$ for $t \in J_{x} \backslash\{i\}$, and further by Corollary 4.7 (ii), we get $I_{y}(e)=\emptyset$, and so $\left(y_{(t)}, x_{(t)}\right)=(1,1)$ for $t \in[k] \backslash J_{x}$. Hence, we have $\varepsilon(x) \in E\left(P_{2}^{\square k}\right)$, and so $E\left(H_{2}\right) \subseteq E\left(P_{2}^{\square k}\right)$. It follows from $\left|E\left(H_{2}\right)\right|=\left|E\left(P_{2}^{\square k}\right)\right|$ that $H_{2}=P_{2}^{\square k}$, as desired.

\subsection{A poset on $\mathcal{C}_{k}$}

In this subsection, we focus on the poset $\left(\mathcal{C}_{k}, \preceq\right)$, and always suppose that $H_{2}$ is a graph with the vertex set $[3]^{k}$. We begin by constructing a graph on the set $[3]^{k}$. Let $\Gamma_{k}$ be the graph with the vertex set $[3]^{k}$ and the edge set

$$
E\left(\Gamma_{k}\right)=\left\{\{x, y\}\left|x, y \in[3]^{k}, x \neq y,\right| x_{(i)}-y_{(i)} \mid \leq 1 \text { for each } i \in[k]\right\} .
$$

Observation 4.13 $\Gamma_{k}=\operatorname{span}\left(\bigcup_{i \in[k]}\left(E\left(C_{i}^{k}\right) \cup E\left(D_{i}^{k}\right)\right)\right)$.

Theorem 4.14 Suppose that $\bar{K}_{[k]} \circ H_{2} \in \mathcal{C}_{k}$.

(i) Then $H_{2} \preceq \Gamma_{k}$.

(ii) If $H_{2} \preceq H_{2}^{\prime} \preceq \Gamma_{k}$, then $\bar{K}_{[k]} \circ H_{2}^{\prime} \in \mathcal{C}_{k}$.

Proof. (i) Combining Proposition 2.4 (i) and Observation 4.13, we have $E\left(H_{2}\right) \subseteq$ $E\left(\Gamma_{k}\right)$, and so $H_{2} \preceq \Gamma_{k}$.

(ii) Observe that $E\left(H_{2}^{\prime}\right) \subseteq E\left(\Gamma_{k}\right), M_{i}\left(H_{2}\right) \subseteq M_{i}\left(H_{2}^{\prime}\right)$ and $N_{i}\left(H_{2}\right) \subseteq N_{i}\left(H_{2}^{\prime}\right)$, where $M_{i}\left(H_{2}\right)$ and $N_{i}\left(H_{2}\right)$ are as refer to (3) and (4), respectively. For each $i \in[k]$, by Observation 4.13 , we have $M_{i}\left(\Gamma_{k}\right)=E\left(C_{i}^{k}\right)$ and $N_{i}\left(\Gamma_{k}\right)=E\left(D_{i}^{k}\right)$, which are $[3]_{i}^{k}(2)$-covering and $S_{i}^{k}$-covering, respectively. Hence, the desired result follows from Proposition 2.4.

Corollary 4.15 For any graph $\bar{K}_{[k]} \circ H_{2} \in \mathcal{C}_{k}$, we have $\left|E\left(H_{2}\right)\right| \leq \frac{7^{k}-3^{k}}{2}$, with equality if and only if $H_{2}=\Gamma_{k}$. 
Proof. Noting that $\left|x_{(i)}-y_{(i)}\right| \leq 1$ if and only if

$$
\left(x_{(i)}, y_{(i)}\right) \in\{(1,1),(1,2),(2,1),(2,2),(2,3),(3,2),(3,3)\},
$$

we have $\mid\left\{(x, y)\left|x, y \in[3]^{k},\right| x_{(i)}-y_{(i)} \mid \leq 1\right.$ for each $\left.i \in[k]\right\} \mid=7^{k}$, which implies that $\left|E\left(\Gamma_{k}\right)\right|=\frac{7^{k}-3^{k}}{2}$. Hence, by Theorem 4.14, we get the desired result.

Corollary 4.16 For $G_{1}, G_{2} \in \mathcal{C}_{k}$, we have $G_{1} \cup G_{2} \in \mathcal{C}_{k}$. In particular, we have

$$
\bigcup_{G \in \mathcal{C}_{k}} G=\bar{K}_{[k]} \circ \Gamma_{k} \in \mathcal{C}_{k}
$$

Proof. Write $G_{1}=\bar{K}_{[k]} \circ H_{2}$ and $G_{2}=\bar{K}_{[k]} \circ H_{2}^{\prime}$. Then $G_{1} \cup G_{2}=\bar{K}_{[k]} \circ\left(H_{2} \cup H_{2}^{\prime}\right)$. Hence, the desired result follows from Theorem 4.14.

The following result is immediate from Corollary 4.16.

Proposition 4.17 The poset $\left(\mathcal{C}_{k}, \preceq\right)$ is a finite join-semilattice with the maximum graph $\bar{K}_{[k]} \circ \Gamma_{k}$.

A graph $H_{2}$ is $k$-minimal if $\bar{K}_{[k]} \circ H_{2} \in \mathcal{C}_{k}$ and $\bar{K}_{[k]} \circ H_{2}^{\prime} \notin \mathcal{C}_{k}$ for $H_{2}^{\prime} \prec H_{2}$.

Remark 5 A graph $H_{2}$ is $k$-minimal if and only if $\bar{K}_{[k]} \circ H_{2}$ is minimal in $\mathcal{C}_{k}$.

To study $k$-minimal graph, we give the following notation for convenience.

Notation 7 Write $X=[3]_{[k]}^{k}(\{2,3\}), Y=[3]_{[k]}^{k}(\{1,3\})$ and

$$
\bar{X}=[3]^{k} \backslash X, \quad \bar{Y}=[3]^{k} \backslash Y, \quad Z=[3]^{k} \backslash(X \cup Y) .
$$

We establish the bounds for the size of a $k$-minimal graph.

Theorem 4.18 If $\mathrm{H}_{2}$ is k-minimal, then

$$
\frac{3^{k}+1}{2} \leq\left|E\left(H_{2}\right)\right| \leq k \cdot\left(3^{k-1}+2^{k-1}\right) .
$$

Proof. For $i \in[k]$, denote by $M_{i}^{\prime}$ (resp. $N_{i}^{\prime}$ ) the set of edges $e$ in $M_{i}\left(H_{2}\right)$ (resp. $\left.N_{i}\left(H_{2}\right)\right)$ such that $M_{i}\left(H_{2}\right) \backslash\{e\}\left(\right.$ resp. $\left.N_{i}\left(H_{2}\right) \backslash\{e\}\right)$ is not a $[3]_{i}^{k}(2)$-covering (resp. an $S_{i}^{k}$-covering), where $M_{i}\left(H_{2}\right)$ (resp. $N_{i}\left(H_{2}\right)$ ) is as refer to (3) (resp. (4)). Then $\left|M_{i}^{\prime}\right| \leq\left|[3]_{i}^{k}(2)\right|=3^{k-1}$ and $\left|N_{i}^{\prime}\right| \leq\left|S_{i}^{k}\right|=2^{k-1}$. Since $H_{2}$ is $k$-minimal, it follows from Proposition 2.4 that $E\left(H_{2}\right)=\bigcup_{i \in[k]}\left(M_{i}^{\prime} \cup N_{i}^{\prime}\right)$, and so

$$
\left|E\left(H_{2}\right)\right|=\left|\bigcup_{i \in[k]}\left(M_{i}^{\prime} \cup N_{i}^{\prime}\right)\right| \leq \sum_{i \in[k]}\left(\left|M_{i}^{\prime}\right|+\left|N_{i}^{\prime}\right|\right) \leq k \cdot\left(3^{k-1}+2^{k-1}\right) .
$$

In the following, we prove the lower bound. Pick any vertex $x \in[3]^{k}$. We shall find an edge $e_{x}$ in $H_{2}$ covering $x$ for the following two cases. 
Case 1. $x=(3, \ldots, 3)$. Then $x \in S_{1}^{k}$. Note that $\bar{K}_{[k]} \circ H_{2} \in \mathcal{C}_{k}$. By Proposition 2.4 (iii), there exists at least one edge $e_{x}$ in $H_{2}$ covering $x$.

Case 2. $x \in \bar{Y}$. Then there is an index $i \in[k]$ with $x_{(i)}=2$. By Proposition 2.4 (ii), there is an edge $e_{x}=\{x, y\}$ in $H_{2}$ such that $y_{(i)}=1$, and so $y \in \bar{X}$.

Observing that $X=(X \cap \bar{Y}) \cup\{(3, \ldots, 3)\}$ and $Z \subseteq \bar{Y}$, we choose two families of edges from the above two cases:

$$
F_{X}=\left\{e_{x} \mid x \in X\right\} \quad \text { and } \quad F_{Z}=\left\{e_{x} \mid x \in Z\right\} .
$$

By Case 2, the edge $e_{x}$ for $x \in X \cap \bar{Y}$ does not cover any other vertices in $X$ except $x$, and so $\left|F_{X}\right|=|X|=2^{k}$. Noting that $Z \subseteq \bar{X}$, we have derived that all of the vertices covered by edges in $F_{Z}$ are in $\bar{X}$, which implies that $F_{X} \cap F_{Z}=\emptyset$. Since each edge covers two vertices, one gets $2\left|F_{Z}\right| \geq|Z|=3^{k}-2^{k+1}+1$ by a short calculation. Consequently, we have

$$
\left|E\left(H_{2}\right)\right| \geq\left|F_{X} \cup F_{Z}\right|=\left|F_{X}\right|+\left|F_{Z}\right| \geq 2^{k}+\frac{3^{k}-2^{k+1}+1}{2}=\frac{3^{k}+1}{2},
$$

as desired.

Remark 6 If $\bar{K}_{[k]} \circ H_{2}$ is minimal in $\mathcal{C}_{k}$, then by the equation (1), Remark 5 and Theorem 4.18, we have

$$
\frac{(2 k+3) \cdot 3^{k-1}+1}{2} \leq\left|E\left(\bar{K}_{[k]} \circ H_{2}\right)\right| \leq 2 k \cdot\left(3^{k-1}+2^{k-2}\right) .
$$

To characterize the lower bound in Theorem 4.18, we define a graph on $[3]^{k}$.

Example 4.19 Define $\mathbb{T}_{k}$ as the graph with the vertex set $[3]^{k}$ and the edge set $E\left(\mathbb{T}_{k}\right)=E_{X} \cup E_{Z}$, where

$$
\begin{aligned}
& E_{X}=\left\{\{x, y\} \mid x \in X, y \in[3]^{k}, x_{(i)}-y_{(i)}=1 \text { for each } i \in[k]\right\}, \\
& E_{Z}=\left\{\{x, y\} \mid x, y \in Z, x_{(i)}=y_{(i)}=3 \text { or }\left\{x_{(i)}, y_{(i)}\right\}=\{1,2\} \text { for each } i \in[k]\right\} .
\end{aligned}
$$

Then $\mathbb{T}_{k}$ is a $k$-minimal graph with size $\frac{3^{k}+1}{2}$.

Proof. We first compute $\left|E\left(\mathbb{T}_{k}\right)\right|$. Since there is a bijection from $X$ to $E_{X}$, one has $\left|E_{X}\right|=|X|=2^{k}$. Each vertex in $Z$ is covered by a unique edge in $E_{Z}$, so $E_{Z}$ is a matching in $Z$, which implies that

$$
\left|E_{Z}\right|=\frac{|Z|}{2}=\frac{3^{k}-2^{k+1}+1}{2} .
$$

Noting that $E_{Z}$ does not cover any vertex in $X$, we have $E_{X} \cap E_{Z}=\emptyset$. Hence, we get $\left|E\left(\mathbb{T}_{k}\right)\right|=\left|E_{X}\right|+\left|E_{Z}\right|=\frac{3^{k}+1}{2}$.

The next thing is to prove $\bar{K}_{[k]} \circ \mathbb{T}_{k} \in \mathcal{C}_{k}$. Take any index $i \in[k]$ and then pick any vertex $x \in[3]_{i}^{k}(2) \cup S_{i}^{k}$. If $x \in X$, let $y$ be a vertex in $[3]^{k}$ such that $y_{(t)}=x_{(t)}-1$ for each $t \in[k]$, then $\{x, y\} \in E_{X} \cap E\left(C_{i}^{k}\right)$ or $E_{X} \cap E\left(D_{i}^{k}\right)$ according to $x \in[3]_{i}^{k}(2)$ 
or $S_{i}^{k}$. If $x \in \bar{X}$, then $x \in Z$, and so $\{x, y\} \in E_{Z} \cap E\left(C_{i}^{k}\right)$, where $y$ is a vertex in $Z$ such that

$$
y_{(t)}= \begin{cases}2, & \text { if } x_{(t)}=1 \\ 1, & \text { if } x_{(t)}=2, \\ 3, & \text { if } x_{(t)}=3\end{cases}
$$

Therefore, we have derived that $M_{i}\left(H_{2}\right)$, as refer to (3), is a $[3]_{i}^{k}(2)$-covering and $N_{i}\left(H_{2}\right)$, as refer to (4), is an $S_{i}^{k}$-covering. Observing that $E_{X} \cup E_{Z} \subseteq \bigcup_{i \in[k]}\left(E\left(C_{i}^{k}\right) \cup\right.$ $\left.E\left(D_{i}^{k}\right)\right)$, we get $\bar{K}_{[k]} \circ H_{2} \in \mathcal{C}_{k}$ from Proposition 2.4.

Finally, we show that $\mathbb{T}_{k}$ is $k$-minimal. By contradiction, if $\mathbb{T}_{k}$ is not $k$-minimal, then there is a graph $H_{2} \prec \mathbb{T}_{k}$ such that $\bar{K}_{[k]} \circ H_{2} \in \mathcal{C}_{k}$, and so $\left|E\left(H_{2}\right)\right|<\left|E\left(\mathbb{T}_{k}\right)\right|=$ $\frac{3^{k}+1}{2}$, contrary to Theorem 4.18 . This contradiction implies that $\mathbb{T}_{k}$ is minimal.

The proof is complete.

We use the following result to characterize the lower bound in Theorem 4.18.

Corollary 4.20 The lower bound in Theorem 4.18 is attained if and only if $\mathrm{H}_{2}=$ $\mathbb{T}_{k}$.

Proof. The "if" implication follows from Example 4.19. To obtain the "only if" implication, let $H_{2}$ be a $k$-minimal graph with size $\frac{3^{k}+1}{2}$. With references to the proof of Theorem 4.18, we have derived from (10) that

$$
E\left(H_{2}\right)=F_{X} \cup F_{Z}, \quad\left|F_{X}\right|=2^{k}=\left|E_{X}\right| \quad \text { and } \quad\left|F_{Z}\right|=\frac{|Z|}{2}=\left|E_{Z}\right| .
$$

It follows from Proposition 2.4 that $F_{X}=E_{X}$ and $F_{Z}=E_{Z}$, as desired.

To characterize the upper bound in Theorem 4.18, we give the following notation.

Notation 8 For $i \in[k]$ and $x \in[3]_{i}^{k}(2) \cup S_{i}^{k}$, denote by $\epsilon_{i}(x)$ the set of edges $e=\left\{x, x^{\prime}\right\}$ such that $x_{(i)}^{\prime}=x_{(i)}-1$ and for each $t \in[k] \backslash\{i\}$, one of the following conditions holds.

(i) If $x \in S_{i}^{k}$, then $x_{(t)}^{\prime}=x_{(t)}$.

(ii) If $x \in X \cap[3]_{i}^{k}(2)$, then

$$
x_{(t)}^{\prime}= \begin{cases}2 \text { or } 3, & \text { if } x_{(t)}=2, \\ 3, & \text { if } x_{(t)}=3 .\end{cases}
$$

(iii) If $x \in \bar{X} \cap[3]_{i}^{k}(2)$, then

$$
x_{(t)}^{\prime}= \begin{cases}1, & \text { if } x_{(t)}=1, \\ 2 \text { or } 3, & \text { if } x_{(t)}=2 \text { or } 3 .\end{cases}
$$

Lemma 4.21 Let $i, j \in[k]$. Pick $x \in[3]_{i}^{k}(2) \cup S_{i}^{k}$ and $y \in[3]_{j}^{k}(2) \cup S_{j}^{k}$. If $(i, x) \neq$ $(j, y)$, then $\epsilon_{i}(x) \cap \epsilon_{j}(y)=\emptyset$. 
Proof. Suppose for the contrary that $\epsilon_{i}(x) \cap \epsilon_{j}(y) \neq \emptyset$. Take $e \in \epsilon_{i}(x) \cap \epsilon_{j}(y)$.

Case 1. $i=j$. Then $x \neq y$ and $e=\{x, y\}$, which implies that $y_{(i)}=x_{(i)}-1$ and $x_{(i)}=y_{(i)}-1$, a contradiction.

Case 2. $i \neq j$ and $x=y$. Write $e=\left\{x, x^{\prime}\right\}$. Then

$$
x_{(i)}^{\prime}=x_{(i)}-1 \quad \text { and } \quad x_{(j)}^{\prime}=x_{(j)}-1 .
$$

Note that $e \in \epsilon_{i}(x)$. If $x \in S_{i}^{k}$ or $X \cap[3]_{i}^{k}(2)$, then $x_{j}^{\prime} \geq x_{j}$, a contradiction. If $x \in \bar{X} \cap[3]_{i}^{k}(2)$, then $\left(x_{(j)}^{\prime}, x_{(j)}\right)=(2,3)$, which implies that $x \in S_{j}^{k}$, and so $x \in X$, a contradiction.

Case 3. $i \neq j$ and $x \neq y$. Then $e=\{x, y\}$, and so

$$
y_{(i)}=x_{(i)}-1 \quad \text { and } \quad y_{(j)}=x_{(j)}+1 .
$$

If $x \in S_{i}^{k}$, then $y_{(j)}=x_{(j)}$, a contradiction. Now suppose $x \in[3]_{i}^{k}(2)$. Then $y_{(i)}=1$ and $\left(x_{(j)}, y_{(j)}\right)=(2,3)$. Noting that $\{x, y\} \in \epsilon_{j}(y)$, we have $y \in S_{j}^{k}$, and so $x_{(i)}=y_{(i)}$, a contradiction.

We accomplish the proof.

Example 4.22 Define

$$
\mathcal{Q}_{k}=\left\{\operatorname{span}\left(\bigcup_{i \in[k]} \bigcup_{x \in[3]_{i}^{k}(2) \cup S_{i}^{k}}\left\{e_{i}(x)\right\}\right) \mid e_{i}(x) \in \epsilon_{i}(x)\right\} .
$$

Then each graph in $\mathcal{Q}_{k}$ is a $k$-minimal graph with size $k \cdot\left(3^{k-1}+2^{k-1}\right)$.

Proof. Choose any graph $H_{2} \in \mathcal{Q}_{k}$. Observe that $\epsilon_{i}(x) \subseteq E\left(C_{i}^{k}\right) \cup E\left(D_{i}^{k}\right)$. Noting that $E\left(H_{2}\right) \cap \epsilon_{i}(x)$ has exactly one edge, as well as the unique edge covering $x$ in $M_{i}\left(H_{2}\right)$ or $N_{i}\left(H_{2}\right)$ according to $x \in[3]_{i}^{k}(2)$ or $S_{i}^{k}$, we deduce that $H_{2}$ is $k$-minimal from Proposition 2.4 and Lemma 4.21. By Lemma 4.21 again, one has

$$
\left|E\left(H_{2}\right)\right|=k \cdot\left(\left|[3]_{i}^{k}(2)\right|+\left|S_{i}^{k}\right|\right)=k \cdot\left(3^{k-1}+2^{k-1}\right),
$$

as desired.

Remark 7 Let $P_{3}$ be the graph with the vertex set $\{1,2,3\}$ and the edge set $\{\{1,2\},\{2,3\}\}$. Let $\mathbb{Q}_{k}$ be the graph obtained from the Cartesian product $P_{3}^{\square k}$ by deleting the edges $\left\{x, x^{\prime}\right\}$ such that there exist indices $i$ and $j$ in $[k]$ with $\left(x_{i}, x_{i}^{\prime}\right)=(2,3)$ and $x_{j}=x_{j}^{\prime}=1$. Then $\mathbb{Q}_{k} \in \mathcal{Q}_{k}$.

We use the following result to characterize the upper bound in Theorem 4.18.

Corollary 4.23 The upper bound in Theorem 4.18 is attained if and only if $\mathrm{H}_{2} \in$ $\mathcal{Q}_{k}$. 
Proof. We get the sufficiency from Example 4.22. To prove the necessity, let $\mathrm{H}_{2}$ be a $k$-minimal graph with size $k \cdot\left(3^{k-1}+2^{k-1}\right)$. With references to the proof of Theorem 4.18, we have derived from (9) that

$$
\left|M_{i}^{\prime}\right|=3^{k-1}=\left|[3]_{i}^{k}(2)\right|, \quad\left|N_{i}^{\prime}\right|=2^{k-1}=\left|S_{i}^{k}\right|,
$$

and the following condition.

(A) All sets $M_{i}^{\prime \prime}$ s and $N_{i}^{\prime \prime}$ s are pairwise non-intersecting.

Take any $x \in[3]_{i}^{k}(2) \cup S_{i}^{k}$. By (11), there is a unique edge $f_{i}(x)$ covering $x$ in $M_{i}^{\prime}$ or $N_{i}^{\prime}$ according to $x \in[3]_{i}^{k}(2)$ or $S_{i}^{k}$. Note that $E\left(H_{2}\right)=\bigcup_{i \in[k]}\left(M_{i}^{\prime} \cup N_{i}^{\prime}\right)$. To get the desired result, we only need to prove $f_{i}(x) \in \epsilon_{i}(x)$.

Suppose for the contrary that $f_{i}(x) \notin \epsilon_{i}(x)$. It is routine to verify that there is an index $t \in[k] \backslash\{i\}$ such that either $\left(x, f_{i}(x)\right) \in[3]_{t}^{k}(2) \times E\left(C_{t}^{k}\right)$ or $\left(x, f_{i}(x)\right) \in$ $S_{t}^{k} \times E\left(D_{t}^{k}\right)$. Then $f_{i}(x) \in M_{t}\left(H_{2}\right)$ or $N_{t}\left(H_{2}\right)$ according to $x \in[3]_{t}^{k}(2)$ or $S_{t}^{k}$. Noting that $M_{t}^{\prime} \subseteq M_{t}\left(H_{2}\right)$ and $N_{t}^{\prime} \subseteq N_{t}\left(H_{2}\right)$, by the definitions of $M_{t}^{\prime}$ and $N_{t}^{\prime}$ in Theorem 4.18, we get $f_{t}(x)=f_{i}(x)$, which contradicts (A). This contradiction completes the proof.

\section{Perfectness-resolvable}

We begin this section by computing the diameters of graphs in $\mathcal{B}_{k}$ or $\mathcal{C}_{k}$.

Proposition 5.1 (i) The diameter of any graph in $\mathcal{B}_{k}$ is 2 or 3.

(ii) The diameter of any graph in $\mathcal{C}_{k}$ is 3,4 or 5.

Proof. (i) Choose any graph $H \in \mathcal{B}_{k}$. Pick $i, j \in[k]$. By Lemma 3.1 (i), we have $d(i, z)=z_{(i)}$ for each $z \in[2]^{k}$, and so

$$
d(i, j) \leq d(i,(1, \ldots, 1))+d(j,(1, \ldots, 1))=1+1=2 .
$$

For distinct vertices $x, y \in[2]^{k}$, there exists a vertex $t \in[k]$ such that $x_{(t)}=1$ or $y_{(t)}=1$. Hence, we have

$$
d(x, y) \leq d(t, x)+d(t, y) \leq 1+2=3 .
$$

Since $d(i,(2, \ldots, 2))=2$, the diameter of $H$ is 2 or 3 .

(ii) An argument similar to the proof of (i) shows that (ii) holds.

Remark 8 (i) The diameters of $K_{[k]} \circ K_{[2]^{k}}$ and $K_{[k]} \circ \mathbb{U}_{2}$ are 2 and 3, respectively.

(ii) The diameters of $\bar{K}_{[k]} \circ \Gamma_{k}, \bar{K}_{[k]} \circ \mathbb{Q}_{2}$ and $\bar{K}_{[k]} \circ \mathbb{T}_{2}$ are 3,4 and 5 , respectively.

Given a graph $G$, the metric dimension of $G$, denoted by $\operatorname{dim}(G)$, is the minimum cardinality of a resolving set of $G$. A metric basis of $G$ is a resolving set of $G$ with cardinality $\operatorname{dim}(G)$. A metric basis of $G$ is perfect if it is a completeness-resolving set. We say that $G$ is perfectness-resolvable if it admits a perfect metric basis. Clearly, a perfectness-resolvable graph is completeness-resolvable. 
Observation 5.2 (i) All paths are perfectness-resolvable.

(ii) A graph in $\mathcal{K}$ is perfectness-resolvable if and only if it is complete.

Proposition 5.3 (i) Let $G$ be a graph in $\mathcal{B}_{k}$. If the diameter of $G$ is 2 , then $G$ is perfectness-resolvable.

(ii) Let $G$ be a graph in $\mathcal{C}_{k}$. If the diameter of $G$ is 3 , then $G$ is perfectnessresolvable.

Proof. By [4, Theorem 1], we have $|V(G)| \leq \operatorname{dim}(G)+d^{\operatorname{dim}(G)}$, where $d$ is the diameter of $G$. Note that $|V(G)|$ is equal to $k+2^{k}$ or $k+3^{k}$ according to $G \in \mathcal{B}_{k}$ or $\mathcal{C}_{k}$. Hence, one has $k \leq \operatorname{dim}(G)$ if the condition in (i) or (ii) holds. Since $[k]$ is a completeness-resolving set of graphs in $\mathcal{B}_{k} \cup \mathcal{C}_{k}$, the two desired results follow.

We conclude the paper by raising the following problem.

Problem 2. Which graphs are perfectness-resolvable?

\section{Acknowledgements}

Feng was supported by the National Natural Science Foundation of China (11701281), the Natural Science Foundation of Jiangsu Province (BK20170817) and the Grant of China Postdoctoral Science Foundation. Ma was supported by the National Natural Science Foundation of China (11801441) and the Young Talent fund of University Association for Science and Technology in Shanxi, China (20190507). Xu was supported by the National Natural Science Foundation of China (61673218) and the Postgraduate Research \& Practice Innovation Program of Jiangsu Province (KYCX18_0376 and KYCX19_0253).

\section{References}

[1] R.F. Bailey and P.J. Cameron, Base size, metric dimension and other invariants of groups and graphs, Bull. Lond. Math. Soc. 43 (2011) 209-242.

[2] Z. Beerliova, F. Eberhard, T. Erlebach, A. Hall, M. Hoffmann, M. Mihal'ák and L.S. Ram, Network discovery and verification, IEEE J. on Selected Areas in Communications 24 (2006) 2168-2181.

[3] J. Cáceres, C. Hernando, M. Mora, I.M. Pelayo, M.L. Puertas, C. Seara and D.R. Wood, On the metric dimension of Cartesian products of graphs, SIAM J. Discrete Math. 21 (2007) 423-441.

[4] G. Chartrand, L. Eroh, M. A. Johnson and O. R. Oellermann, Resolvability in graphs and the metric dimension of a graph, Discrete Appl. Math. 105 (2000) 99-113.

[5] V. Chvátal, Mastermind, Combinatorica 3 (1983) 325-329.

[6] F. Harary and R.A. Melter, On the metric dimension of a graph, Ars Combinatoria 2 (1976) 191-195. 
[7] Z. Jiang and N. Polyanskii, On the metric dimension of Cartesian powers of a graph, J. Combin. Theory Ser. A 165 (2019) 1-14.

[8] S. Khuller, B. Raghavachari and A. Rosenfeld, Landmarks in graphs, Discrete Appl. Math. 70 (1996) 217-229.

[9] A. Sebő and E. Tannier, On metric generators of graphs, Math. Oper. Res. 29 (2004) 383-393.

[10] P.J. Slater, Leaves of trees, Congr. Numerantium 14 (1975) 549-559. 